\title{
Langmuir
}

This document is confidential and is proprietary to the American Chemical Society and its authors. Do not copy or disclose without written permission. If you have received this item in error, notify the sender and delete all copies.

\section{Structural studies of non-ionic dodecanol ethoxylates at the oil-water interface: the effect of increasing head group size}

\begin{tabular}{|r|l|}
\hline Journal: & Langmuir \\
\hline Manuscript ID: & Draft \\
\hline Manuscript Type: & Article \\
\hline Date Submitted by the Author: & $\mathrm{n} / \mathrm{a}$ \\
\hline Complete List of Authors: & $\begin{array}{l}\text { Zarbakhsh, Ali; Queen Mary, University of London, Chemistry } \\
\text { Campana, Mario; University of Manchester, School of Physics and } \\
\text { Astronomy } \\
\text { Webster, John; STFC Rutherford appleton laboratory, isis facility }\end{array}$ \\
\hline
\end{tabular}

\section{SCHOLARONE ${ }^{\text {m }}$ \\ Manuscripts}




\title{
Structural studies of non-ionic dodecanol ethoxylates at the oil-water interface: the effect of increasing head group size
}

\author{
Mario Campana ${ }^{1}$, John. R. P. Webster ${ }^{2}$ and Ali Zarbakhsh ${ }^{3^{*}}$ \\ ${ }^{1}$ School of Physics and Astronomy, University of Manchester, Oxford Road, Manchester, M13 9PL, \\ United Kingdom. \\ ${ }^{2}$ ISIS Neutron Facility, Science and Technology Facilities Council, Rutherford Appleton Laboratory, Harwell \\ Science and Innovation Campus, Didcot, OX11 0QX, United Kingdom. \\ ${ }^{3}$ School of Biological \& Chemical Sciences, Queen Mary, University of London, Joseph Priestley Building, \\ Mile End Road, London E1 4NS, United Kingdom. \\ *Author to whom correspondence should be addressed. Email: a.zarbakhsh@qmul.ac.uk
}

\begin{abstract}
The conformation of charged surfactants at the oil-water interface was recently reported. With the aim to assess the role of the head group size on the conformation of the adsorbed layer, we have extended these studies to a series of non-ionic dodecanol ethoxylate surfactants $\left(C_{12} E_{n}\right.$, ethylene oxide units $n$ from 6 to 12). The study was performed using neutron reflectometry to enable maximum sensitivity to buried interfaces. Similarly to charged surfactants, the interface was found to be broader and rougher compared to the air-water interface. Irrespectively of the head group size, the tail group region was found to assume a staggered conformation. The conformations of the head group were found to be significantly different compared to the air-water interface, moving from a globular to an almost fully extended conformation at the oil-water interface. The stretching of the head groups is attributed to the presence of some hexadecane oil molecules, which may penetrate all the way to this region. It is proposed here that the presence of the oil, which can efficiently solvate the surfactant tail groups, plays a key role in the conformation of the adsorbed layer and is responsible for the broadening of the interface.
\end{abstract}




\section{Introduction}

The conformation of surfactants at the oil-water interface is generally very different from the airwater interface. At the air-water interface, when a surfactant molecule in the aqueous phase reaches the water surface it partially interacts with the air phase. The only favourable interactions left available for the hydrophobic part of the surfactant are Van der Waals interactions between different tail groups. For this reason, the hydrophobic part of the adsorbed layer tends to be confined to a specific region in contact with the air phase where it assumes a relatively tightly packed conformation, i.e. a well-ordered monolayer [1,2]

At the oil-water interface, on the other hand, oil molecules can efficiently solvate surfactant tail groups. When a surfactant molecule in water reaches the interface, its tail group can establish Van der Waals interactions both with other surfactant tail groups and with oil molecules. As a result, the tail group region can assume a more relaxed and energetically favourable conformation and the whole adsorbed layer results in a broader structure. The effect of the presence of charges in the surfactant head group on the structure of the adsorbed layer was recently investigated both for cationic and zwitterionic surfactants [3-5]. The results obtained suggested a multilayer structure, where a staggered conformation was observed.

With this study we aimed to extend the knowledge of surfactants adsorption at the oil-water interface to non-ionic surfactants. Particularly, the effect of an increasing size of the head group on the structure of the adsorbed layer was investigated. A series of non-ionic dodecanol ethoxylate surfactants $\left(\mathrm{C}_{12} \mathrm{E}_{\mathrm{n}}\right)$ was chosen for this study. By increasing the number of ethylene oxide units, $n$, from 6 to 12, the size of the head group can be steadily increased. Alkyl ethoxylates are extensively used in detergency, shampoo, fabric softening, cosmetics and pharmacy. Their interfacial adsorption and aggregation in solution have a key role in their use in many product formulations. Much is known about bulk properties of dodecanol ethoxylates such as cloud point as a function of increasing $n$ [6], or properties of mixed micelles containing $\mathrm{C}_{12} \mathrm{E}_{\mathrm{n}}[7,8]$. The addition of $\mathrm{C}_{12} \mathrm{E}_{\mathrm{n}}$ to SDS solutions efficiently reduces the solubilisation of zein proteins, thus reducing the extent to which the detergent formulations will provoke adverse skin reactions [9]. Interfacial behaviour at the solid-water interface has been reported at a number of different substrates such as graphite [10], silicon [11] and cellulose [12].

Besides their extensive use, there were two reasons for choosing dodecanol ethoxylates for this study: 
1. The structural conformation of dodecanol ethoxylates at the air-water interface was fully characterised in the 90s. In a series of a neutron reflectivity experiments the size of the ethoxylate head group was gradually increased from $n=1$ to 12 . The interface was investigated for a series of concentrations above and below the CMC and the effects of the increasing head group on the structural conformation was studied [13-17]. Extensive knowledge of the system at the air-water interface is essential to understand how the head group affects the surfactant conformation at the oil-water interface.

2. As the number of ethylene glycol units increases from $n=1$ to $n=12$, the thickness of the tail group region at the air-water interface, as determined by neutron reflectivity, varies very little. The thickness varies between 11 and $13 \AA$, suggesting a tilted conformation of the tail groups $\left(\theta \sim 40^{\circ}\right)$. Hence, it is the size of the head group that plays a key role on the conformation at the air-water interface. One would expect a similar behaviour on the final conformation at the oil-water interface.

Neutron Reflectivity (NR) has been extensively used in the past few years for the investigation of surfactant adsorption at the oil-water [3-5]. The short wavelength of neutrons allow the structural resolution normal to the interface on the length scale of few $\AA$. NR also having the important advantage to employ isotopic substitution to achieve large and tunable contrast between chemically discrete entities and allows precise structural determination. This paper concentrates on detailed aspects of the structure of non-ionic surfactants at the oil-water interface.

\section{Materials}

Dodecanol ethoxylate surfactants $\left(\mathrm{C}_{12} \mathrm{E}_{\mathrm{n}}\right)$ with progressively increasing head group size $(n=6,8$ and 12) were used in this study. Surfactants were synthesised by Dr R. K. Thomas and co-workers from University of Oxford and were available as fully hydrogenous. Hydrogenous hexadecane was purchased from Aldrich and was purified by passing it seven times through an alumina column prior to use. Hexadecane- $\mathrm{d}_{34}$ was obtained from Cambridge Isotope Laboratories $(>98 \% \mathrm{D})$ and was used with no further purification. $\mathrm{D}_{2} \mathrm{O}$ was obtained from Fluorochem $(>99.9 \%$ D). All solutions and isotopic mixtures were prepared by mass. Contrast-matched silicon hexadecane contains approximately $35 \%$ d-hexadecane mixed with h-hexadecane (CMSi oil).

\section{Methods}


Although most ionic surfactants are practically insoluble in oil, this is not always the case for nonionic surfactants, mainly because of the absence of charges in the head group. It has already been reported that $\mathrm{C}_{12} \mathrm{E}_{\mathrm{n}}$ surfactants present a higher solubility in hydrocarbons than in water [17]. As a result, partition processes between oil and water occur, thus lowering the aqueous concentration of the surfactant. When neutron reflectivity is used to probe the buried liquid-liquid interface the volume of the hydrophobic phase is much smaller than that of the aqueous phase (with the deployed setting $\sim 15 \mu$ of hexadecane, $\sim 25 \mathrm{ml}$ of aqueous phase). Therefore, partition processes only cause a minor decrease of surfactant aqueous concentration.

Neutron reflectometry is a technique sensitive to neutron refractive index profile normal to an interface, $N b(z)$, averaged for the whole sample region [18]. In absence of strong neutron absorbers such as cadmium or boron, the neutron refractive index can be written as:

$$
n(\lambda) \approx 1-\frac{\lambda^{2}}{2 \pi} N b
$$

$N$ is the atomic number density and $b$ the coherent scattering length. The multiple $N b$, referred to as the scattering length density, varies linearly with the volume fraction composition:

$$
N b \approx \sum_{j} \Phi_{j} N b_{j}
$$

where $\Phi_{j}$ is the volume fraction and $(\mathrm{Nb})_{j}$ the scattering length density of component $j$, respectively. Possibly the main advantage of neutron reflectivity is that the scattering length $b$ varies in a random fashion within different elements in the periodic table; particularly $b$ varies within different isotopes. Above all, $b$ is substantially different between hydrogen $\left(b_{H}=-3.74 \mathrm{fm}\right)$ and deuterium $\left(b_{D}=6.67 \mathrm{fm}\right)$. This difference is at the basis of the methodology called contrast variation. By changing the isotopic ratio, the $N b$ of the molecule can be adjusted to match that of other components in the system or to maximise the difference in refractive index.

The methodology developed by Zarbakhsh et al. [3] was used for these experiments. To avoid drastic attenuation of the neutron beam upon trespassing the oil phase, a thin layer of hexadecane was deposited by spin-coating onto a silicon block which had been rendered hydrophobic by coupling of trimethylchlorosilane. The film was frozen in place, and the sample cell assembled with the oil still frozen. The aqueous surfactant solution was slowly injected in the sample cell, 
sandwiching the oil film between the silicon substrate and the aqueous phase. Once the cell was deemed to be bubble free, the hexadecane film was allowed to melt. A circulating water bath was used to cool the cell at $\sim 278 \mathrm{~K}$ while assembling the sample cell and maintain a thermostated temperature of $298 \pm 1 \mathrm{~K}$ throughout the whole neutron reflectivity measurement.

The thick film approximation gives the reflectivity R for a sufficiently thick oil film [19]:

$$
R=R_{1}+\frac{A R_{2}\left(1-R_{1}\right)^{2}}{1-A R_{1} R_{2}}
$$

$\mathrm{R}_{1}$ is the reflectivity from the silicon-hexadecane interface (generally minimised by using CMSi oil), and $\mathrm{R}_{2}$ the reflectivity from the hexadecane-aqueous solution interface. The attenuation factor A takes into account the loss of intensity upon the beam crossing the oil film twice:

$$
A=\exp \left(\frac{-2 \chi d_{o i l}}{\sin \theta_{o i l}}\right)
$$

The attenuation coefficient $\chi$ was experimentally determined prior to the experiment, $d_{o i l}$ is the thickness of the oil film and $\theta_{\text {oil }}$ is the incidence angle of the neutron beam in the oil phase [3].

Since a large number of measurements was required to fully characterise three surfactants at different concentrations, a series of neutron reflectivity investigations were planned and three different reflectometers were used for this study. $\mathrm{C}_{12} \mathrm{E}_{6}$ was analysed using FIGARO, at ILL, Grenoble, France [20], $\mathrm{C}_{12} \mathrm{E}_{8}$ was analysed using INTER, at ISIS, Didcot, UK [21] and $\mathrm{C}_{12} \mathrm{E}_{12}$ was analysed using SURF, also at ISIS [22]. The three instruments are time-of-flight reflectometers and the available wavelength range changes significantly between them. The incident angle was therefore varied to cover a most suitable $Q$ range. The incident angle was $3.82^{\circ}$ for $\mathrm{C}_{12} \mathrm{E}_{6}, 2.3^{\circ}$ for $\mathrm{C}_{12} \mathrm{E}_{8}$ and $1.5^{\circ}$ for $\mathrm{C}_{12} \mathrm{E}_{12}$. In all measurements $\mathrm{D}_{2} \mathrm{O}$ was chosen as the aqueous subphase to exploit the contrast with the fully hydrogenous surfactant and maximise the sensitivity to the adsorbed layer. CMSi oil was used to minimise the $\mathrm{R}_{1}$ part of the reflectivity.

\section{Results}

A series of reflectivity profiles were measured for all surfactants for a series of concentrations above and below the recorded $\mathrm{CMC}$ at the air-water interface. $\mathrm{CMC}$ values for the three surfactants at the air-water interface are given in Table 1. Reflectivity profiles for all concentrations are shown in Figure 1 for $\mathrm{C}_{12} \mathrm{E}_{6}$, Figure 2 for $\mathrm{C}_{12} \mathrm{E}_{8}$ and Figure 3 for $\mathrm{C}_{12} \mathrm{E}_{12}$. Because the attenuation of the neutron beam upon traversing the oil phase is wavelength-dependent, the data analysis must be 
carried out in wavelength $(\lambda)$. The data are, however, conventionally presented in $Q(\mathrm{Q}=(4 \pi \sin \theta$ )/ $\lambda$ ) for clarity.

Table 1. CMC values at the air-water interface for the surfactants used.

\begin{tabular}{|c|c|}
\hline Surfactant & CMC / mM \\
\hline $\mathrm{C}_{12} \mathrm{E}_{6}[14]$ & 0.080 \\
\hline $\mathrm{C}_{12} \mathrm{E}_{8}[15]$ & 0.091 \\
\hline $\mathrm{C}_{12} \mathrm{E}_{12}[17]$ & 0.125 \\
\hline
\end{tabular}

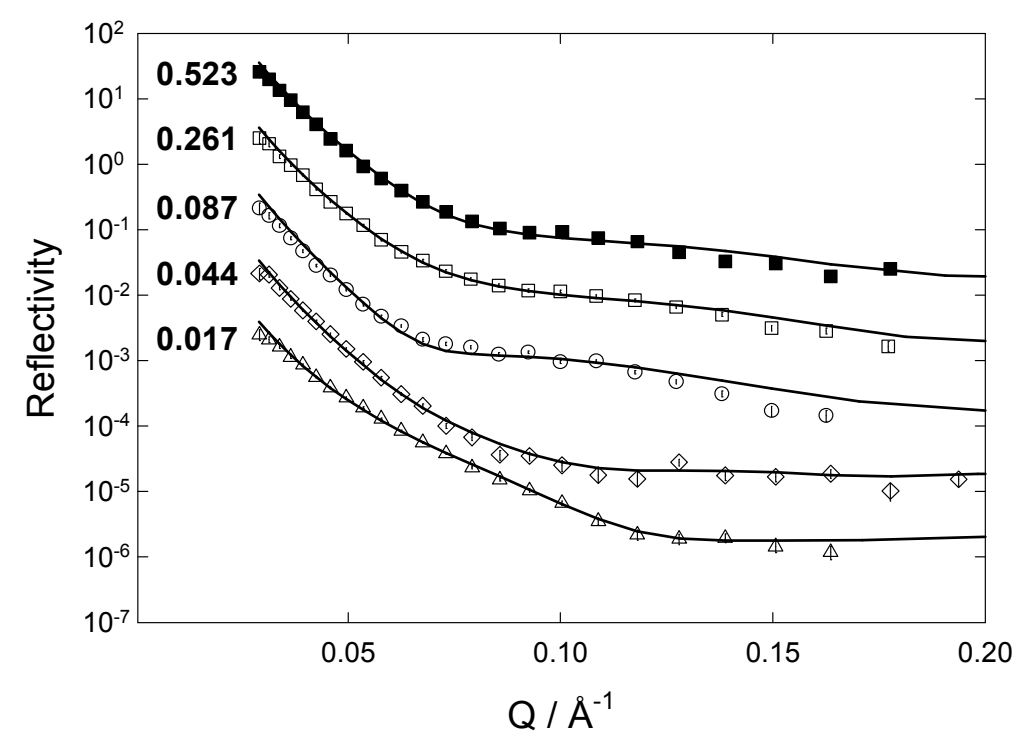

Figure 1. Reflectivity profiles for a series of $h-C_{12} E_{6}$ at the $C M S i$ oil- $D_{2} O$ interface, solid lines correspond to the fit to the data. The concentration is shown in $\mathrm{mM}$ units. Profiles are shifted by a factor of $\times 10$ for the purpose of clarity. 


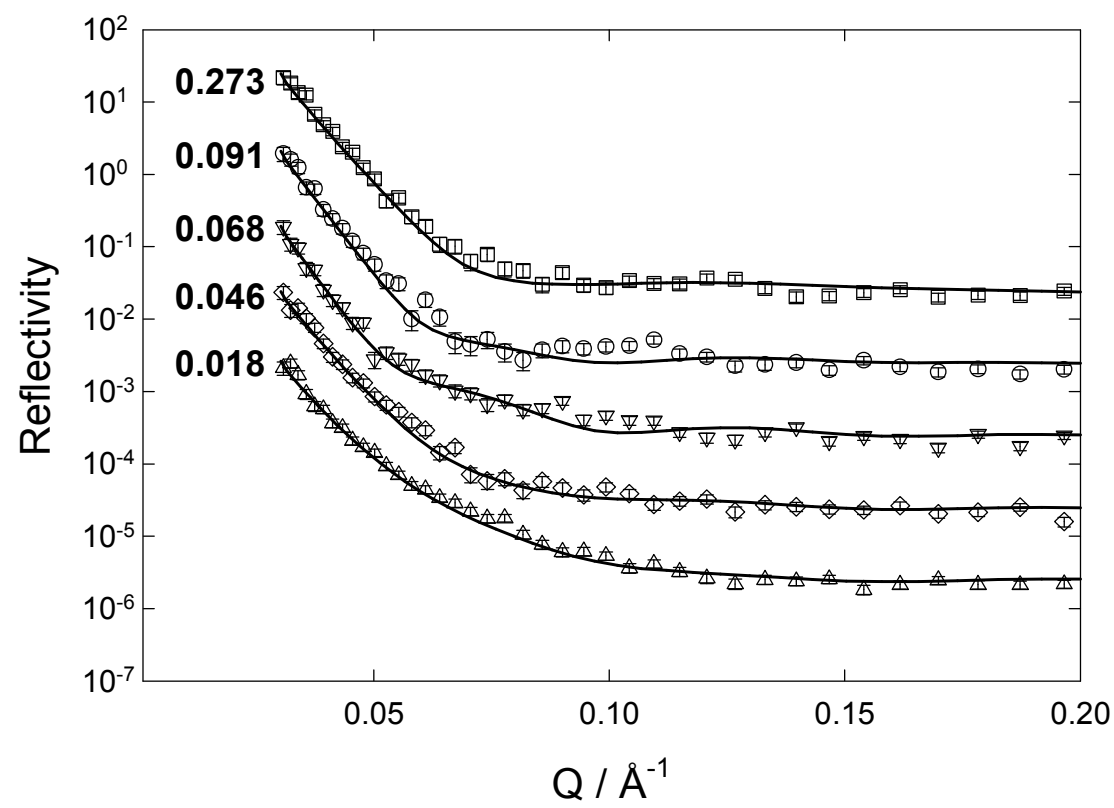

Figure 2. Reflectivity profiles for a series of $h-C_{12} E_{8}$ at the $C M S i$ oil- $D_{2} O$ interface, solid lines correspond to the fit to the data. The concentration is shown in $\mathrm{mM}$ units. Profiles are shifted by a factor of $\times \mathbf{1 0}$ for the purpose of clarity.

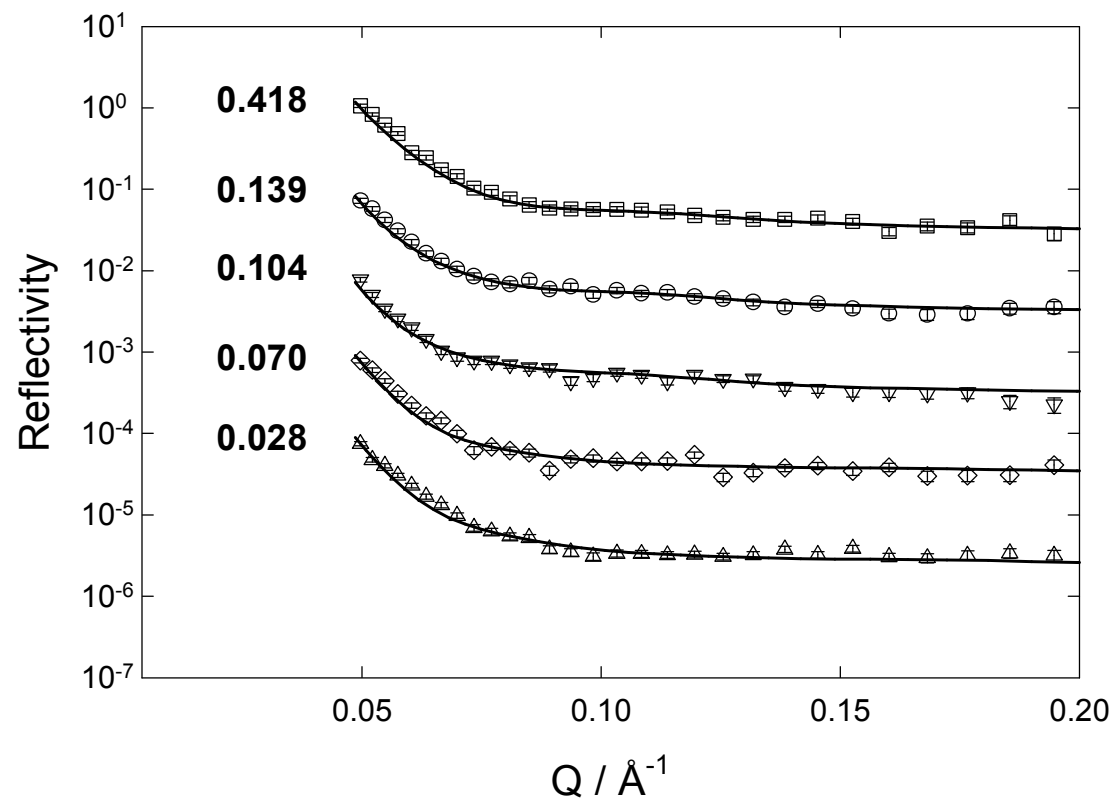

Figure 3. Reflectivity profiles for a series of $h-C_{12} E_{12}$ at the $C M S i$ oil- $D_{2} O$ interface, solid lines correspond to the fit to the data. The concentration is shown in $\mathrm{mM}$ units. Profiles are shifted by a factor of $\times \mathbf{1 0}$ for the purpose of clarity. 
All reflectivity profiles were fitted to a three layer model. The thickness of the first layer, in contact with the oil phase, was found to be constant ( $22 \pm 2 \AA)$ regardless of the increasing size of the head group. On the other hand, the thickness of the second, intermediate layer increased with increasing head group size. A small decrease in thickness of the second layer was observed for $\mathrm{C}_{12} \mathrm{E}_{12}$ when the concentration decreases to low values $\left(\left[\mathrm{C}_{12} \mathrm{E}_{12}\right]<0.104 \mathrm{mM}\right)$. A third layer, with $N b$ close to that of $\mathrm{D}_{2} \mathrm{O}$, was required to model the reflectivity data. The thickness of this layer increased from $\mathrm{C}_{12} \mathrm{E}_{6}$ to $\mathrm{C}_{12} \mathrm{E}_{8}$ and no further changes were observed upon increasing the head group to 12 ethylene oxide units. The thickness of this layer decreased for $\mathrm{C}_{12} \mathrm{E}_{6}$ with decreasing concentration $\left(\left[\mathrm{C}_{12} \mathrm{E}_{6}\right]<0.261 \mathrm{mM}\right)$.

Changes in interlayer roughness between the oil phase and the first layer, between the first and the second layer, and between the third layer and the $\mathrm{D}_{2} \mathrm{O}$, affect very little the reflectivity profiles. Conversely, small variations in the roughness between the second and the third layer significantly affect the quality of the fitting. Roughness between the second and the third layer increases considerably between $\mathrm{C}_{12} \mathrm{E}_{6}$ and $\mathrm{C}_{12} \mathrm{E}_{8}$ and a further increase is observed between $\mathrm{C}_{12} \mathrm{E}_{8}$ and $\mathrm{C}_{12} \mathrm{E}_{12}$. Roughness between the second and the third layer is particularly relevant in the fitting procedure and will henceforth be referred to as $\sigma_{3}$.

All the fitting parameters are shown in Table 2, 3 and 4, for $\mathrm{C}_{12} \mathrm{E}_{6}, \mathrm{C}_{12} \mathrm{E}_{8}$ and $\mathrm{C}_{12} \mathrm{E}_{12}$ respectively. The corresponding $N b$ profiles are shown in Figure 4, Figure 5 and Figure 6 for $\mathrm{C}_{12} \mathrm{E}_{6}, \mathrm{C}_{12} \mathrm{E}_{8}$ and $\mathrm{C}_{12} \mathrm{E}_{12}$.

Table 2. Fitting parameters for $\mathrm{C}_{12} \mathrm{E}_{6}$ at the contrast-matched silicon hexadecane- $\mathrm{D}_{2} \mathrm{O}$ interface.

High concentration

\begin{tabular}{|c|c|c|c|}
\hline Concentration / mM & 0.523 & 0.261 & \\
\hline $\begin{array}{c}\text { Layer thickness } \\
\AA( \pm 2)\end{array}$ & \multicolumn{2}{|c|}{$\mathrm{Nb} \times 10^{-6} / \AA^{-2}$} & Roughness / $\AA$ \\
\hline Hexadecane & 2.07 & 2.78 & 2 \\
\hline 22 & 3.10 & 2.88 & 2 \\
\hline 25 & 5.93 & 5.94 & 5 \\
\hline 35 & & 6.35 & 2 \\
\hline $\mathrm{D}_{2} \mathrm{O}$ & \multicolumn{2}{|c}{} & \\
\hline
\end{tabular}




\section{Low concentration}

\begin{tabular}{|c|c|c|c|c|}
\hline Concentration / mM & 0.087 & 0.044 & 0.017 & Roughness / $\AA$ \\
\hline $\begin{array}{c}\text { Layer thickness } \\
\AA( \pm 2 \AA)\end{array}$ & \multicolumn{3}{|c|}{$\mathrm{Nb} \times 10^{-6} / \AA^{-2}$} & - \\
\hline Hexadecane & 3.20 & 3.43 & 3.88 & 2 \\
\hline 22 & 3.32 & 4.98 & 5.89 & 5 \\
\hline 25 & 5.96 & 6.03 & 6.13 & 2 \\
\hline 24 & & 6.35 & & 5 \\
\hline $\mathrm{D}_{2} \mathrm{O}$ & & & \\
\hline
\end{tabular}


Table 3. Fitting parameters for $\mathrm{C}_{12} \mathrm{E}_{8}$ at the contrast-matched silicon hexadecane- $\mathrm{D}_{2} \mathrm{O}$ interface.

\begin{tabular}{|c|c|c|c|c|c|c|}
\hline Concentration / mM & 0.273 & 0.091 & 0.068 & 0.046 & 0.018 & \\
\hline $\begin{array}{c}\text { Layer thickness } \\
\AA( \pm 2)\end{array}$ & \multicolumn{5}{|c|}{$\mathrm{Nb} \times 10^{-6} / \AA^{-2}$} & $\begin{array}{c}\text { Roughness } \\
\AA\end{array}$ \\
\hline Hexadecane & & 2.07 & - \\
\hline 22 & 2.46 & 2.87 & 3.24 & 3.36 & 3.66 & 2 \\
\hline 30 & 3.29 & 3.95 & 4.33 & 4.59 & 5.08 & 2 \\
\hline 42 & 5.70 & 5.76 & 5.77 & 6.09 & 6.15 & 11 \\
\hline $\mathrm{D}_{2} \mathrm{O}$ & & 6.35 & 2 \\
\hline
\end{tabular}

Table 4. Fitting parameters for $C_{12} E_{12}$ at the contrast-matched silicon hexadecane- $D_{2} O$ interface.

High concentration

\begin{tabular}{|c|c|c|c|c|}
\hline Concentration / mM & 0.418 & 0.139 & 0.104 & Roughness / $\AA$ \\
\hline $\begin{array}{c}\text { Layer thickness } \\
\AA( \pm 2)\end{array}$ & \multicolumn{3}{|c|}{$\mathrm{Nb} \times 10^{-6} / \AA^{-2}$} & - \\
\hline Hexadecane & & 2.07 & 2 \\
\hline 22 & 2.33 & 2.47 & 2.54 & 2 \\
\hline 42 & 2.57 & 2.86 & 2.98 & 13 \\
\hline 42 & 5.64 & 5.73 & 5.77 & 2 \\
\hline $\mathrm{D}_{2} \mathrm{O}$ & & 6.35 & & \\
\hline
\end{tabular}

Low concentration

\begin{tabular}{|c|c|c|c|}
\hline Concentration / mM & 0.070 & 0.028 & \\
\hline $\begin{array}{c}\text { Layer thickness } \\
\AA( \pm 2)\end{array}$ & \multicolumn{2}{|c|}{$\mathrm{Nb} \times 10^{-6} / \AA^{-2}$} & Roughness $/ \AA$ \\
\hline Hexadecane & 2.07 & 2.96 & 2 \\
\hline 22 & 4.44 & 4.49 & 2 \\
\hline 35 & 6.13 & 6.13 & 2 \\
\hline 42 & \multicolumn{2}{|c|}{6.35} & \\
\hline $\mathrm{D}_{2} \mathrm{O}$ & & & \\
\hline
\end{tabular}




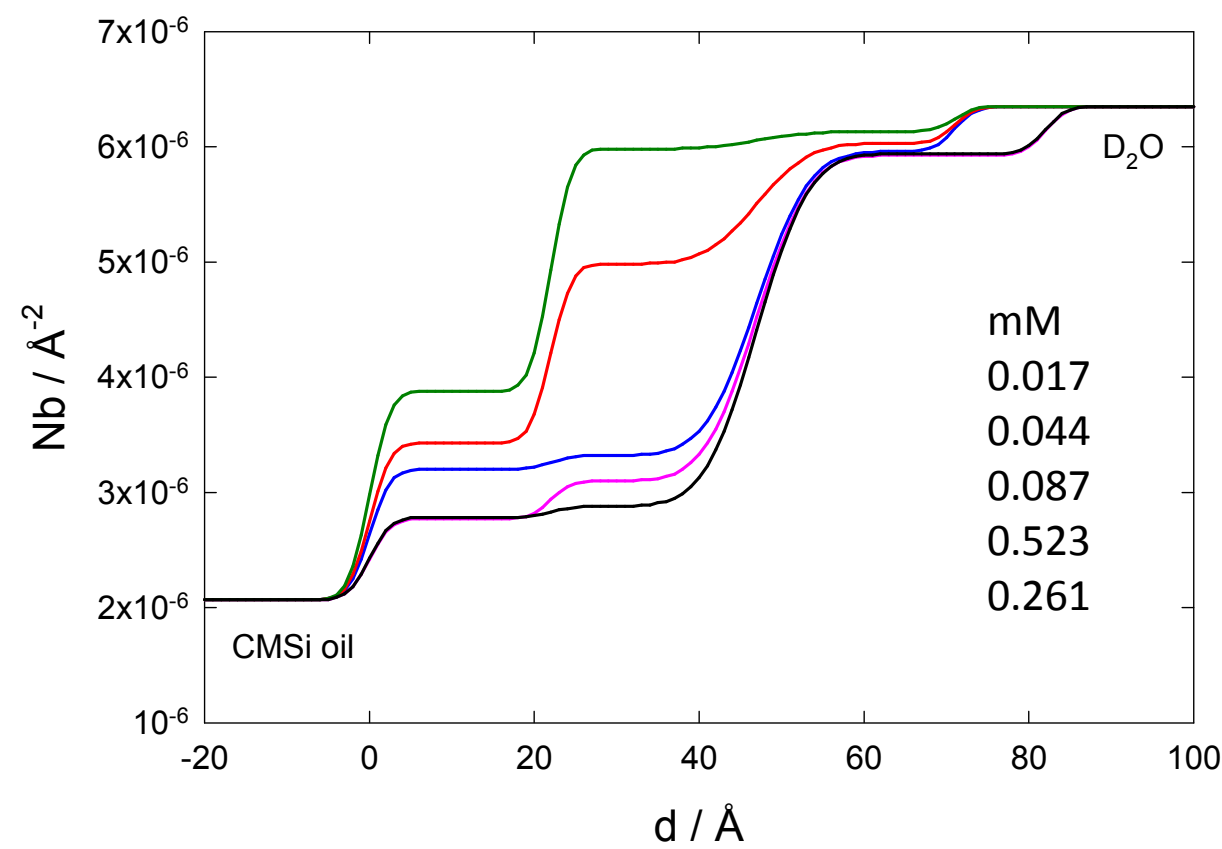

Figure 4. $N b$ profiles for a series of $\mathrm{C}_{12} \mathrm{E}_{6}$ at the $\mathrm{CMSi}$ hexadecane- $\mathrm{D}_{2} \mathrm{O}$ interface.

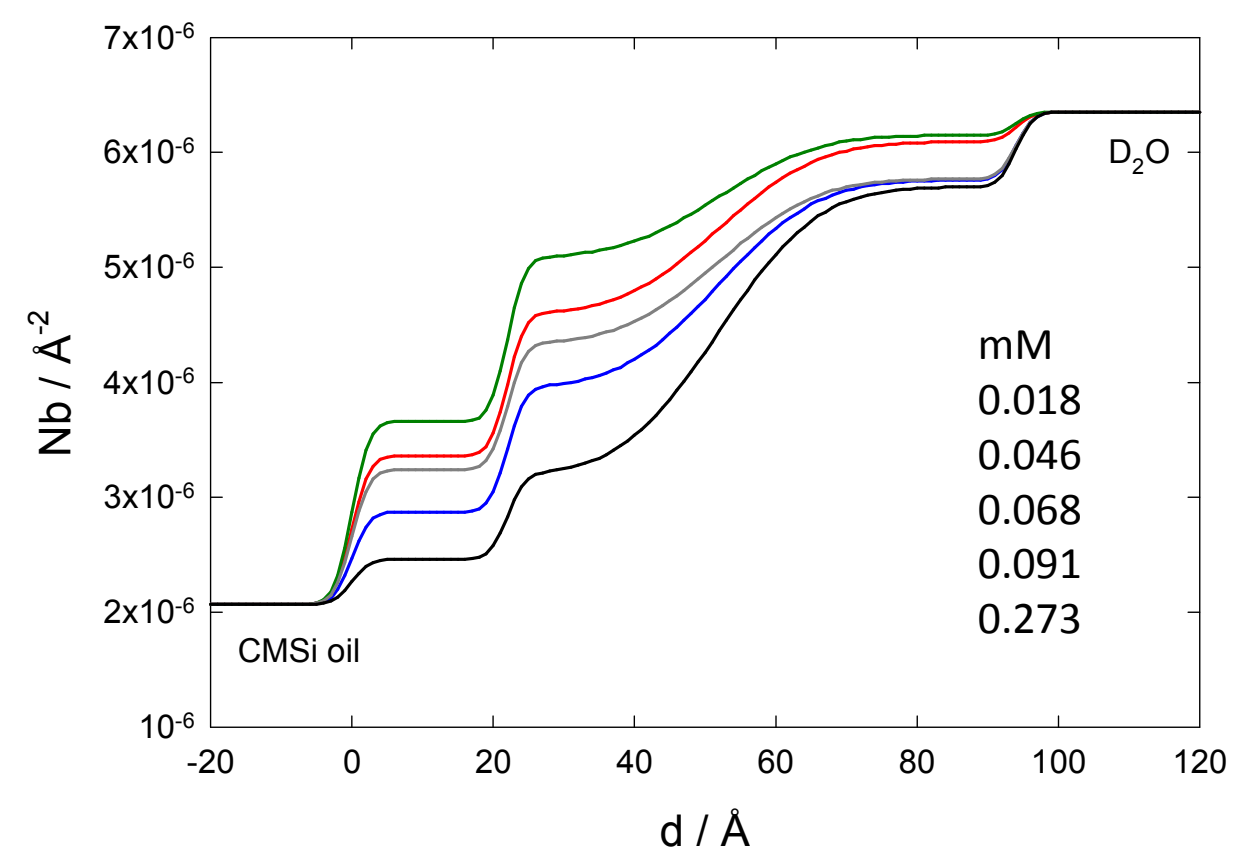

Figure 5. $N b$ profiles for a series of $\mathrm{C}_{12} \mathrm{E}_{8}$ at the $\mathrm{CMSi}$ hexadecane- $\mathrm{D}_{2} \mathrm{O}$ interface. 


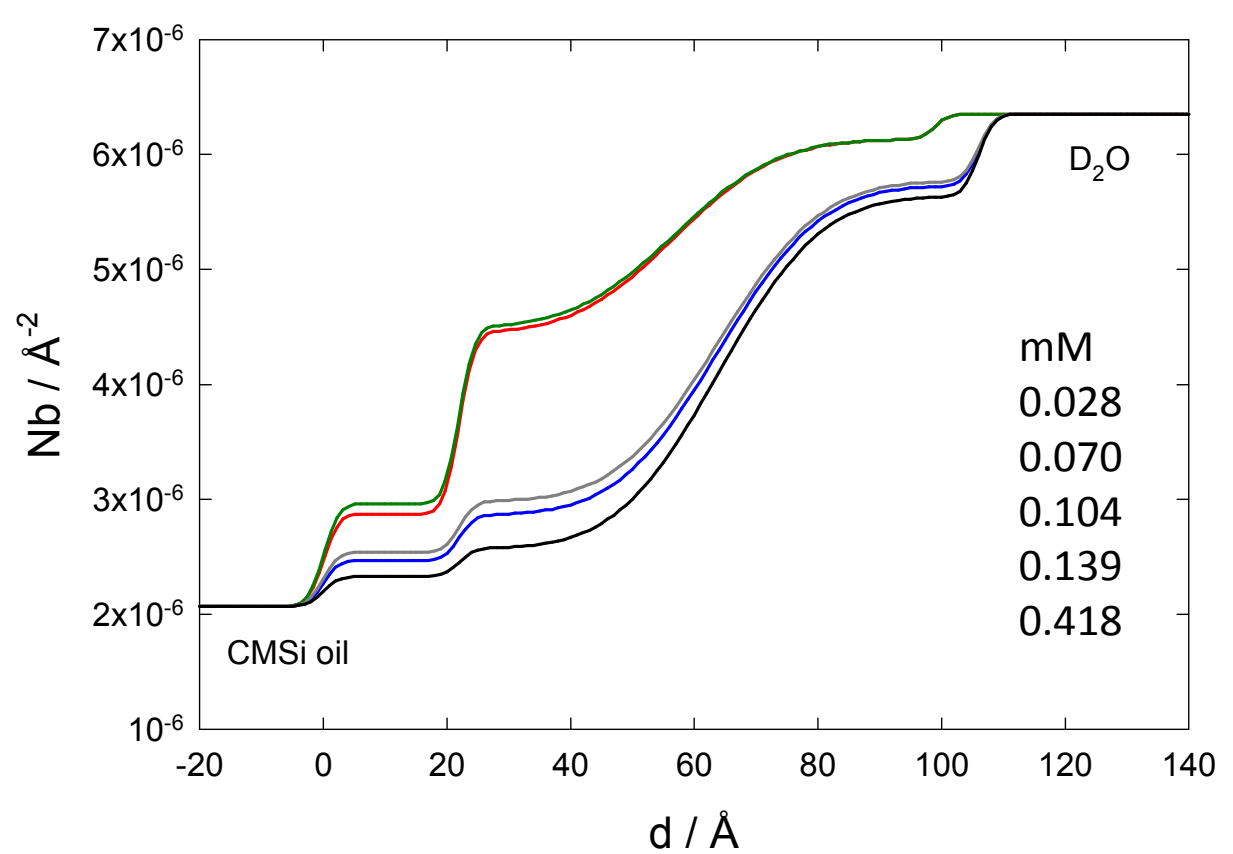

Figure 6. $N b$ profiles for a series of $\mathrm{C}_{12} \mathrm{E}_{12}$ at the $\mathrm{CMSi}$ hexadecane- $\mathrm{D}_{2} \mathrm{O}$ interface.

\section{Discussion}

The interface was divided into three layers which represent three distinct regions of the interfacial area. The first layer, with a thickness of $22 \pm 2 \AA$ for all contrasts, represents the region where the tail group inserts into the oil phase. The thickness is broader compared to the fully extended dodecyl chain length $(\sim 16.3 \AA)$, indicating a relatively staggered conformation. This is not surprising given the rougher nature of the oil-water interface. The chain solvation by the hexadecane molecules prevents the perfect alignment of the tail groups, playing a key role in maintaining the staggered conformation in this region of the interface. Such broadening of the tail group region at the oil-water interface was also observed for the zwitterionic $\mathrm{C}_{16} \mathrm{PC}$ molecules [4], indicating that solvation of the tail group occurs regardless of the presence or absence of charges in the head group. The $N b$ of the first layer is in all cases higher than that of the oil and the surfactant, indicating the presence of water $\left(\mathrm{D}_{2} \mathrm{O}\right)$ in the region. The $N b$ of the first layer decreases as a function of increasing head group size, therefore the presence (volume fraction) of $\mathrm{D}_{2} \mathrm{O}$ in this region must also decrease. The phenomenon might be related to surface activity: the surfactant with the smaller head group $\left(\mathrm{C}_{12} \mathrm{E}_{6}\right)$ is more surface active and leads to better intermixing between oil and water. The increase in head group size leads to a decrease in the surface activity of the 
compound; hence the progressively worse intermixing between oil and water and the reduced presence of water $\left(\mathrm{D}_{2} \mathrm{O}\right)$ in the layer.

The second layer contains mostly surfactant head groups. The thickness of this layer increases as a function of increasing head group size, which is to be expected as the head group gets bulkier. The thickness of the region is comparable to the length of the fully extended head group for all surfactants. It must be stated, however, that a reduction in layer thickness was observed for $\mathrm{C}_{12} \mathrm{E}_{12}$ at low concentrations and as a result the thickness deviates from the fully extended length of the ethoxylated fragment.

Figure 7 shows the thickness of the head group region both at the air-water $(\bullet)$ and at the oil-water (o) interface. At the air-water interface the increase in thickness above $n=4$ was limited because of globular shape assumed by the ethoxylated region upon folding.

As a staggered conformation was already observed for the tail group region, it is not realistic to expect a perfectly aligned orientation for the head groups either. However, the staggered conformation alone cannot account for the significant increase in thickness compared to the airwater interface. A possible explanation for such an increase in thickness compared to the air-water interface is that at the oil-water interface the head group assumes a comparably less globular and more extended conformation, with no appreciable folding of the head group region.

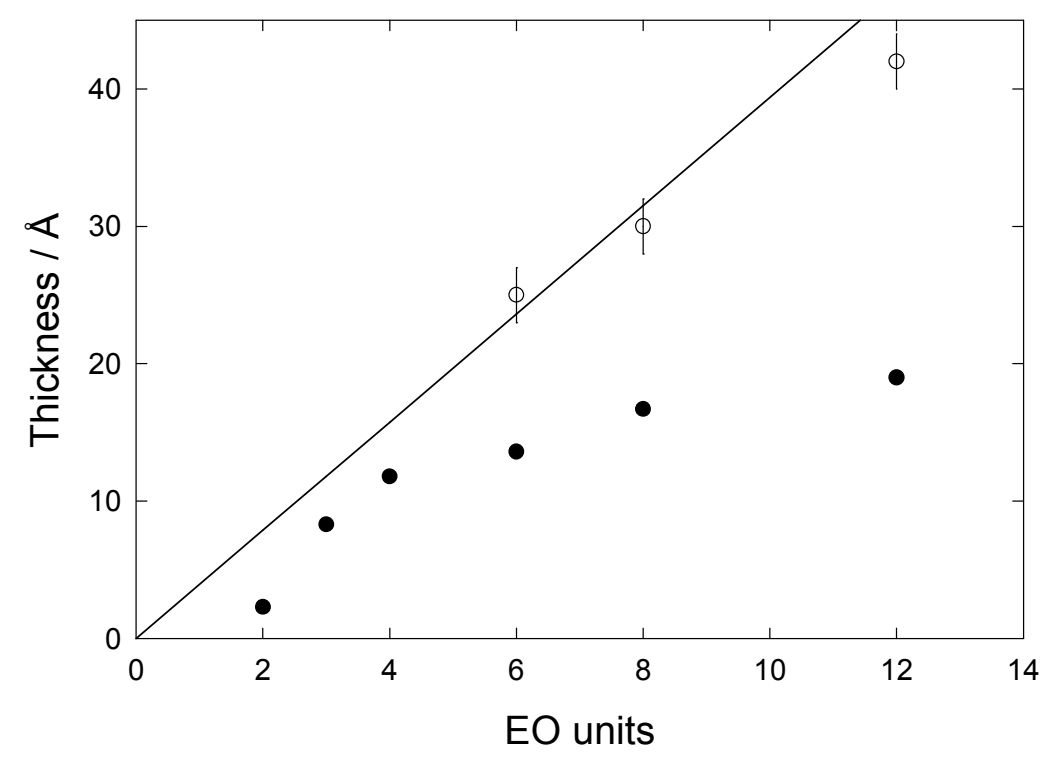

Figure 7. Thickness of the head group region as a function of head group size at the air-water $(\bullet)$ and the oil-water interface (o). The straight line indicates the length of the fully extended head group. 
The third layer, the one in contact with $\mathrm{D}_{2} \mathrm{O}$, represents a diffuse underlying structure. The $N b$ of the layer is very close to $\mathrm{D}_{2} \mathrm{O}$ in all cases, indicating that little surfactant is adsorbed in this region. It must be stressed that since the $\triangle N b$ between the diffuse layer and $\mathrm{D}_{2} \mathrm{O}$ is very small, the sensitivity of neutron reflectivity to this interfacial region is rather limited and the discussion regarding the structure of this layer is bound to be highly speculative. For all surfactants, as the concentration decreases below the $\mathrm{CMC}$, the amount of surfactant in the underlying structure decreases (the $\mathrm{Nb}$ of the underlying structure increases to almost that of $\mathrm{D}_{2} \mathrm{O}$ ). $\mathrm{C}_{12} \mathrm{E}_{\mathrm{n}}$ surfactants form Gibbs monolayers where molecules from the bulk keep exchanging with those adsorbed at the interface. The underlying structure, adjacent to the adsorbed primary monolayer, represents the interfacial region where the exchange between the bulk phase and the monolayer takes place. This region could be interpreted as a surfactant-enriched area of the bulk phase in the vicinity of the interface; a necessary pathway for surfactant molecules to reach the interface. This hypothesis is also suggested by the observation that the $N b$ of the third layer consistently decreases as the size of the head group increases. In fact, surfactants with larger head groups present higher CMC, so that the concentration of free monomers in solution increases steadily with increasing head group size, hence increasing the concentration in the surfactant-enriched layer and slightly diminishing its $\mathrm{Nb}$.

Interlayer roughness plays an important role in the data fitting for $\mathrm{C}_{12} \mathrm{E}_{\mathrm{n}}$ surfactants. No interlayer roughness was required between the oil phase and layer 1, between layer 1 and layer 2 and between layer 3 and $\mathrm{D}_{2} \mathrm{O}$. A roughness of $2 \AA$ was used solely to smooth the steps in the $N b$ profiles in Figure 4, Figure 5 and Figure 6 and to improve the visual representation. Conversely, the roughness between the second layer and the diffuse layer $\left(\sigma_{3}\right)$ increases significantly from 5 to $11 \AA$ when moving from 6 to 8 ethylene oxide units in the head group. A further small increase was observed for $n=12\left(\sigma_{3}=13 \AA\right)$. The increased roughness indicates that the interdigitation between the primary monolayer and the diffuse region increases. The increased intermixing between the two regions could be caused by a combination of two factors:

1. Increased disorder in the head group region of the primary monolayer with increasing head group size. This is not unlikely as it has already been observed at the air-water interface [17].

2. As the size of the head group increases, the CMC increases, and so does the concentration of free monomers in the aqueous phase. The surfactant molecules become more hydrophilic 
and progressively less surface active, consequently the interaction between the surfactant molecules and the interface becomes less favourable. The turnover between the primary monolayer and the diffuse region becomes much faster with increasing head group size, therefore it becomes progressively more difficult using neutron reflectivity to discern neatly between the two regions. An increase in roughness is then required to represent the imperfectly neat separation between the two regions.

The structure of the primary monolayer in direct contact with the oil phase significantly differs from that at the air-water interface. At the air-water interface the tail region was constant and thinner than the fully extended length of the alkyl region, so that the tail groups were assuming a tilted conformation. At the oil-water interface the thickness of the tail group region also remains constant as a function of increasing head group size, but the thickness is now larger than the fully extended alkyl chain length. This effect is caused by the presence of hexadecane, which is now solvating the alkyl chains so that tail-tail interactions are reduced. This results in a staggered conformation of the surfactant molecules where steric repulsion between the tail groups is much reduced. A similar increase in layer thickness for the tail group region when moving from the air-water to the oil-water interface was already observed for $\mathrm{C}_{16} \mathrm{PC}$ surfactants and it was associated with a significant reduction in tilt angle [4].

Penfold et al., suggested a possible increase of water presence in the tail group region with increasing head group size at the air-water interface [22]. The opposite seems to happen at the oilwater interface: the $\mathrm{Nb}$ of the tail group region decreases with increasing head group size, clearly indicating that the presence of $\mathrm{D}_{2} \mathrm{O}$ in the layer decreases as the head group gets bulkier.

Of particular interest is the increase of layer thickness for the head group region as a function of increasing head group size. It was shown earlier that the thickness of the head group region at the air-water interface increases linearly up to $n=4$; after that, the increase is not as pronounced because of globular conformation assumed by the head group. At the oil-water interface, on the other hand, the thickness of the head group region seems to increase linearly with increasing size (Figure 7). The much faster increase observed at the oil-water interface can be caused by the head group assuming an almost fully extended conformation. One possible explanation for the extended conformation could be as follows. If some hexadecane molecules were to be present in this region, they could form van der Waals interactions with the hydrocarbon part of the head group preventing it from folding and assuming a globular conformation. This is clearly highly speculative and more detailed contrast schemes would be required to prove it. However, some support for the validity of this theory comes from the presence of the diffuse underlying structure. The hydrocarbon tail 
groups of the surfactants in the underlying structure require a partially hydrophobic anchoring surface in order to adsorb onto the primary monolayer. As the diffuse region was not observed at the air-water interface, the surfactant head group alone is clearly not able to provide a suitable partially hydrophobic surface. At the oil-water interface, the presence of hexadecane molecules intermixed with the head groups could provide a higher degree of hydrophobicity to the head group region, hence the presence of the underlying structure.

The overall conformation of the primary monolayer is much more extended at the oil-water interface than it is at the air-water interface. The possible presence of hydrophobic hexadecane molecules would provide solvation of the surfactant tail groups and the hydrophilic head groups would also have some hexadecane molecules associated. Such solvation would also justify the far more stretched conformation of the adsorbed monolayer observed compared to the air-water interface. The presences of the oil layer seems to efficiently solvate the surfactant tail groups and pull the adsorbed surfactant molecules towards the more hydrophobic phase. This effect could clearly not be observed at the air-water interface as no solvation can be provided by air. To some extent, air can be considered as a "passive" hydrophobic medium. At the air-water interface, air behaves as a hydrophobic medium but it is clearly not capable of establishing any interactions with the substrates present at the interface. When a surfactant molecule in the aqueous phase reaches the interface it cannot interact with the air phase and the only favourable interactions left available for the hydrophobic part of the surfactant are van der Waals interactions between different tail groups. For this reason, the hydrophobic part of the adsorbed layer tends to be confined to a specific region in contact with the air phase where it is forced to form a relatively well ordered structure. At the oilwater interface, on the other hand, oil molecules can efficiently solvate surfactant molecules. When a surfactant molecule in water reaches the interface, its tail group can establish van der Waals interactions both with other surfactant tail groups and with oil molecules. As a result, the tail group region can assume a more relaxed and energetically favourable conformation and the whole adsorbed layer results in a broader structure. For this reason, the oil surface can be considered as an "active" hydrophobic medium.

\section{Conclusion}

The conformation of $\mathrm{C}_{12} \mathrm{E}_{\mathrm{n}}$ surfactants at the hexadecane-water interface was studied as a function of increasing head group size using neutron reflectivity. It was found that the interface can be divided into two main regions: a rough, staggered monolayer in contact with the oil phase and a diffuse region, extending towards the aqueous phase. The conformation of the head group 
significantly changes compared to the air-water interface, moving from a globular to an almost fully extended conformation at the oil-water interface. We believe that an important role in the stretching of the head groups could be attributed to the presence of hexadecane molecules in this region. The presence of oil molecules in the head group region also could be responsible for the existence of a diffuse underlying structure, which was not observed at the air-water interface. It was believed that the interfacial roughening observed at the oil-water interface for ionic and zwitterionic surfactants was mainly caused by the necessity to accommodate the charges in the head groups $[3,4,5]$. In this paper we have shown that a broader and rougher adsorbed layer is also observed for non-ionic $\mathrm{C}_{12} \mathrm{E}_{\mathrm{n}}$ surfactants at the oil-water interface. Since these surfactants do not carry any charges in the head group, the broadening of the interface must be attributed to the presence of the oil phase, which efficiently solvates the adsorbed layer. 
References:

1. Penfold J., Thomas R. K.; J. Phys.: Condens. Matter, 1990, 2, 1369.

2. Denton A.R., Gray C.G., Sullivan D.E.; Chem. Phys. Lett, 1994, 219, 310.

3. Zarbakhsh A., Querol A., Bowers J., Webster J. R. P.; Faraday Discuss., 2005, 129, 155.

4. Zarbakhsh A., Querol A., Bowers J., Yaseen M., Lu J. R., Webster J. R. P.; Langmuir, 2005, 21,11704 .

5. Zarbakhsh A., Webster J. R. P., Eames J.; Langmuir, 2009, 25, 3953.

6. Sharma K. S., Patil S. P., Rakshit A. K.; Colloid Surface A, 2003, 219, 67.

7. Wang Z. N., Li G. Z., Mu J. H., Zhang W. X.; J. Surfactants Deterg., 2002, 5, 39

8. Zhang H., Li Y., Dubin P., Kato T.; J. Colloid Interf. Sci., 1996, 183, 546.

9. Moore P. N., Puvvada S., Blankschtein D.; Langmuir, 2003, 19, 1009.

10. Patrick H. N., Warr G. G., Manne S., Aksay I. A.; Langmuir, 1997, 13, 4349.

11. Desbene P. L., Portet F., Treiner C.; J. Colloid Interf. Sci., 1997, 190, 350.

12. Singh S. K., Notley S. N.; J. Phys. Chem. B, 2010, 114, 14977.

13. Lu J. R., Lee E. M., Thomas R. K., Penfold J., Flitsch S. L.; Langmuir, 1993, 9, 1352.

14. Lu J. R., Li X. Z., Thomas R. K., Staples E. J., Tucker I., Penfold J.; J. Phys. Chem., 1993, 97, 8012;.

15. Lu J. R., Li Z. X., Thomas R. K., Staples E. J., Thompson L., Tucker I., Penfold J.; J. Phys. Chem., 1994, 98, 6559.

16. Lu J. R., Hromadova M., Thomas R. K., Penfold J.; Langmuir, 1993, 9, 2417.

17. Lu J. R., Su T. J., Li Z. X., Thomas R. K., Staples E. J., Tucker I., Penfold J.; J. Phys. Chem. $B, \mathbf{1 9 9 7 , 1 0 1 , 1 0 3 3 2 .}$

18. Lu J. R., Li Z. X., Thomas R. K., Penfold J.; J. Chem. Soc., Faraday Trans., 1996, 92, 403.

19. Dickinson E., Horne D. S., Phipps J. S., Richardson R. M.; Langmuir, 1993, 9, 242.

20. Campbell R. A., Wacklin H. P., Sutton I., Cubitt R., Fragneto G.; Eur. Phys. J. Plus, 2011, $126,107$.

21. Webster J. R. P., Langridge S., Dalgliesh R. M., Chalrton T. R.; Eur. Phys. J. Plus, 2011, $126,112$.

22. Penfold J., Richardson R. M., Zarbakhsh A., Webster J. R. P., Bucknall D. G., Rennie A. R., Jones R. A. L., Cosgrove T., Thomas R. K., Higgins J. S., Fletcher P. D. I., Dickinson E., Roser S. J., McLure I. A., Hillman A. R., Richards R. W., Staples E. J., Burgess A. N., Simister E. A., White J. W.; J. Chem. Soc., Faraday Trans., 1997, 93, 3899.

23. Penfold J., Thomas R. K.; Phys. Chem. Chem. Phys., 2002, 4, 2648. 


\title{
TOC
}

\section{Structural studies of non-ionic surfactants at the oil-water interface}

\author{
Mario Campana ${ }^{1}$, John. R. P. Webster ${ }^{2}$ and Ali Zarbakhsh ${ }^{1 *}$
}

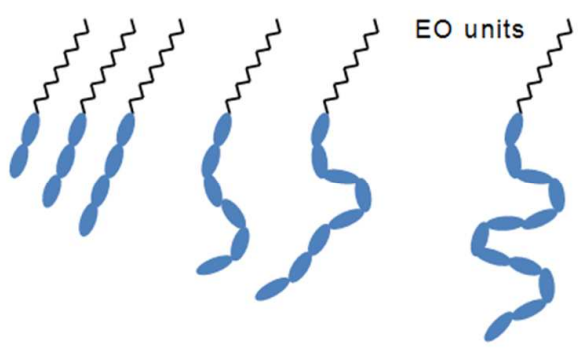

31

32

33

34

35

36

37

38

39

40

41

42

43

44

45

46

47

48

49

50

51

52

53

54

55

56

57

58

59

60 


\title{
TOC
}

\section{Structural studies of non-ionic surfactants at the oil-water interface}

\author{
Mario Campana ${ }^{1}$, John. R. P. Webster ${ }^{2}$ and Ali Zarbakhsh ${ }^{1 *}$
}

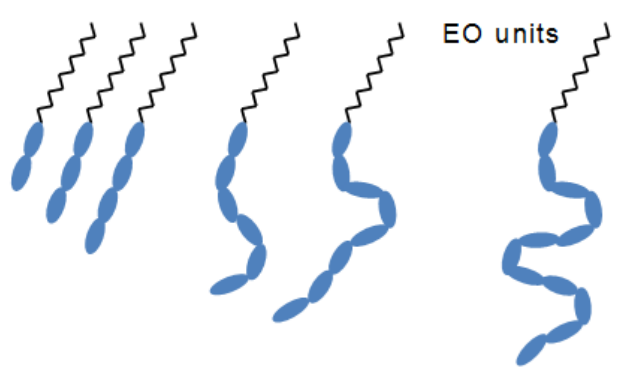

